\title{
Local risk-minimization for Barndorff-Nielsen and Shephard models with volatility risk premium
}

\author{
Takuji Arai*
}

August 22, 2018

\begin{abstract}
We derive representations of local risk-minimization of call and put options for Barndorff-Nielsen and Shephard models: jump type stochastic volatility models whose squared volatility process is given by a nonGaussian Ornstein-Uhlenbeck process. The general form of BarndorffNielsen and Shephard models includes two parameters: volatility risk premium $\beta$ and leverage effect $\rho$. Arai and Suzuki [1] dealt with the same problem under constraint $\beta=-\frac{1}{2}$. In this paper, we relax the restriction on $\beta$; and restrict $\rho$ to 0 instead. We introduce a Malliavin calculus under the minimal martingale measure to solve the problem.
\end{abstract}

Keywords: Local risk-minimization, Barndorff-Nielsen and Shephard models, Stochastic volatility models, Malliavin calculus, Lévy processes.

\section{Introduction}

Local risk-minimization (LRM, for short) for Barndorff-Nielsen and Shephard models (BNS model, for short) is discussed. Here LRM is a very well-known quadratic hedging method of contingent claims for incomplete financial markets. On the other hand, BNS models are stochastic volatility models suggested by Barndorff-Nielsen and Shephard [2], [3]. It is known that some stylized facts of financial time series are captured by BNS models. The square volatility process $\sigma^{2}$ of a BNS model is given as an Ornstein-Uhlenbeck process driven by a subordinator without drift, that is, a nondecreasing pure jump Lévy process. Thus, $\sigma^{2}$ is a jump process given as a solution to the following stochastic differential equation (SDE, for short):

$$
d \sigma_{t}^{2}=-\lambda \sigma_{t}^{2} d t+d H_{\lambda t}, \quad \sigma_{0}^{2}>0,
$$

*Department of Economics, Keio University, e-mail:arai@econ.keio.ac.jp 
where $\lambda>0, H$ is a subordinator without drift. Now, we denote by $S$ the underlying asset price process. The general form of $S$ is given by

$$
S_{t}=S_{0} \exp \left\{\int_{0}^{t}\left(\mu+\beta \sigma_{s}^{2}\right) d s+\int_{0}^{t} \sigma_{s} d W_{s}+\rho H_{\lambda t}\right\},
$$

where $S_{0}>0, \mu, \beta \in \mathbb{R}, \rho \leq 0, W$ is a 1-dimensional Brownian motion. The last term $\rho H_{\lambda t}$ accounts for the leverage effect; and $\beta \sigma_{s}^{2}$ is called the volatility risk premium, which is considered as the compensation required by investors holding volatile assets. From the view of (2.1) below, the volatility risk premium is vanished when $\beta=-\frac{1}{2}$. So that, $\beta$ would take a value greater than or equal to $-\frac{1}{2}$. For more details on BNS models, see Cont and Tankov [4] and Schoutens [9].

Our purpose is to obtain representations of LRM of call and put options for BNS models under constraint $\rho=0$ and no constraint on $\beta$. On the other hand, Arai and Suzuki [1] studied the same problem under constraint $\beta=-\frac{1}{2}$ and no constraint on $\rho$. That is, they dealt with the case where volatility risk premium is not taken into account. To the contrary, we will treat BNS models with volatility risk premium. In other words, we relax the restriction on $\beta$. Instead, we restrict $\rho$ to 0 , which induces the continuity of $S$. Then, $S$ is written as

$$
S_{t}=S_{0} \exp \left\{\int_{0}^{t}\left(\mu+\beta \sigma_{s}^{2}\right) d s+\int_{0}^{t} \sigma_{s} d W_{s}\right\} .
$$

Actually, the continuity of $S$ makes the problem easy to deal with. To calculate LRM, we need to consider the minimal martingale measure (MMM, for short). When $S$ is continuous, the subordinator $H$ remains a Lévy process even under the MMM. On the other hand, the generalization of $\beta$ makes the problem complicated. When $\beta=-\frac{1}{2}$, the density process $Z$ of the MMM is given as a solution to an SDE with the Lipschitz continuity. Thus, as shown in [1], Z has the Malliavin differentiability, which played a vital role in [1]. However, this property is not generalized to the case of $\beta \neq-\frac{1}{2}$. Hence, we need to take a different approach from [1]. In order to overcome this difficulty, making the best of the fact that the Lévy property of $H$ is preserved, we innovate a Malliavin calculus under the MMM. As a result, we can calculate LRM without attention to the property of $Z$.

To our best knowledge, except for [1], there is only one preceding research on LRM for BNS models: Wang, Qian and Wang [13]. Besides they treated the problem under the same parameter restrictions as ours, although they did not use Malliavin calculus. However, their discussion seems to be inaccurate mathematically.

Outline of this paper is as follows. A precise model description and standing assumptions are given in Section 2. In Subsections 2.1 -2.3, we define LRM, the MMM and a Malliavin derivative, respectively. Our main results are provided in Section 3; and conclusions will be given in Section 4 . 


\section{Preliminaries}

We consider a financial market model in which only one risky asset and one riskless asset are tradable. For simplicity, we assume that the interest rate is given by 0 . Let $T>0$ be the finite time horizon. The fluctuation of the risky asset is described as a process $S$ given by (1.1). We consider a complete probability space $(\Omega, \mathcal{F}, \mathbb{P})$ with a filtration $\mathbb{F}=\left\{\mathcal{F}_{t}\right\}_{t \in[0, T]}$ as the underlying space. Suppose that $\mathbb{F}$ is generated by $W_{t}$ and $H_{\lambda t}$; and satisfies the usual condition, that is, $\mathbb{F}$ is right continuous, and $\mathcal{F}_{0}$ contains all null sets of $\mathbb{P}$. The asset price process $S$ given in (1.1) is a solution to the following SDE:

$$
d S_{t}=S_{t-}\left\{\mu d t+\left(\beta+\frac{1}{2}\right) \sigma_{t}^{2} d t+\sigma_{t} d W_{t}\right\}
$$

Denoting $A_{t}:=\int_{0}^{t} S_{s-}\left[\mu+\left(\beta+\frac{1}{2}\right) \sigma_{s}^{2}\right] d s$ and $M_{t}:=S_{t}-S_{0}-A_{t}$, we have $S_{t}=S_{0}+M_{t}+A_{t}$, which is the canonical decomposition of $S$. Further, we denote $L_{t}:=\log \left(S_{t} / S_{0}\right)$ for $t \in[0, T]$, that is,

$$
L_{t}=\mu t+\beta \int_{0}^{t} \sigma_{s}^{2} d s+\int_{0}^{t} \sigma_{s} d W_{s}
$$

Defining $J_{t}:=H_{\lambda t}$, we denote by $N$ the Poisson random measure of $J$, that is, we have $J_{t}=\int_{0}^{\infty} x N([0, t], d x)$. Denoting by $v$ the Lévy measure of $J$, we have that $\widetilde{N}(d t, d x):=N(d t, d x)-v(d x) d t$ is the compensated Poisson random measure. Remark that $N$ and $v$ are defined on $[0, T] \times(0, \infty)$ and $(0, \infty)$, respectively; and $v(d x)=\lambda v^{H}(d x)$, where $v^{H}$ is the Lévy measure of $H$. Moreover, Proposition 3.10 of [4] implies

$$
\int_{0}^{\infty}(x \wedge 1) v(d x)<\infty .
$$

We need to impose the following standing assumptions on $v$ as in [1]. As stated in Remark 2.2 below, the standing assumptions do not exclude representative examples of BNS models, although parameters are restricted.

Assumption 2.1 (A1) The Léry measure $v$ is absolutely continuous with respect to the Lebesgue measure on $(0, \infty)$.

(A2) There exists a $\kappa>0$ such that

- $\kappa>\left[2\left(\beta+\frac{1}{2}\right)^{+}+1\right] \mathcal{B}(T)$,

- $\kappa \geq\left(\beta+\frac{1}{2}\right)^{2} \mathcal{B}(T)$, and

- $\int_{1}^{\infty} e^{2 \kappa x} v(d x)<\infty$,

where $\mathcal{B}(t):=\int_{0}^{t} e^{-\lambda s} d s=\frac{1-e^{-\lambda t}}{\lambda}$ for $t \in[0, T]$. 
Remark 2.2 1. When $\beta=-\frac{1}{2}$, (A2) is equivalent to the existence of $\varepsilon>0$ such that $\int_{0}^{\infty} e^{(2+\varepsilon) \mathcal{B}(T) x} v(d x)<\infty$. In [1] dealing with the case of $\beta=-\frac{1}{2}$, $\int_{0}^{\infty} e^{2 \mathcal{B}(T) x} v(d x)<\infty$ is assumed in their Assumption 2.2, which is almost same as the above (A2) for $\beta=-\frac{1}{2}$.

2. We do not need to assume conditions corresponding to the second condition of Assumption 2.2 in [1], which ensures the positivity of the density of the MMM defined below, since the MMM becomes a probability measure automatically in our setting.

3. Condition (A2) ensures $\int_{0}^{\infty} x^{2} v(d x)<\infty$, which means $\mathbb{E}\left[J_{T}^{2}\right]<\infty$. In addition, we have $\mathbb{E}\left[e^{2 \kappa J_{T}}\right]<\infty$ by Proposition 3.14 of [4].

4. Condition (A1) guarantees Assumption Z1 in Nocolato and Venardos [6], which we need in the proof of Lemma 2.9 below.

5. Assumption 2.1 does not exclude two representative examples of $\sigma^{2}$, "IG-OU" and "Gamma-OU". "IG-OU" is the case where $v^{H}$ is given as

$$
v^{H}(d x)=\frac{a}{2 \sqrt{2 \pi}} x^{-\frac{3}{2}}\left(1+b^{2} x\right) e^{-\frac{1}{2} b^{2} x} \mathbf{1}_{(0, \infty)}(x) d x,
$$

where $a>0$ and $b>0$. The invariant distribution of $\sigma^{2}$ follows an inverseGaussian distribution with $a>0$ and $b>0$. Then $\sigma^{2}$ is called an IG-OU process. If

$$
\frac{b^{2}}{2}>2\left\{\left[2\left(\beta+\frac{1}{2}\right)^{+}+1\right] \vee\left(\beta+\frac{1}{2}\right)^{2}\right\} \mathcal{B}(T),
$$

then Assumption 2.1 is satisfied. Next, "Gamma-OU" is the case where the invariant distribution of $\sigma^{2}$ is given by a Gamma distribution with $a>0$ and $b>0$. In this case, $v^{H}$ is described as

$$
v^{H}(d x)=a b e^{-b x} \mathbf{1}_{(0, \infty)}(x) d x .
$$

As well as the IG-OU case, Assumption 2.1] is satisfied if

$$
b>2\left\{\left[2\left(\beta+\frac{1}{2}\right)^{+}+1\right] \vee\left(\beta+\frac{1}{2}\right)^{2}\right\} \mathcal{B}(T) .
$$

For more details on this topic, see also [6] and [9].

\subsection{Local risk-minimization}

In this subsection, we define LRM. To this end, we define the SC condition firstly; and show that $S$ satisfies it under Assumption 2.1 $S$ is said to satisfy the SC condition, if the following three conditions hold: 
(a) $\left\|[M]_{T}^{1 / 2}+\int_{0}^{T}\left|d A_{S}\right|\right\|_{L^{2}(\mathbb{P})}<\infty$.

(b) Defining a process $\Lambda_{t}:=\frac{1}{S_{t-}} \frac{\mu+\left(\beta+\frac{1}{2}\right) \sigma_{t}^{2}}{\sigma_{t}^{2}}$, we have $A=\int \Lambda d\langle M\rangle$.

(c) The mean-variance trade-off process $K_{t}:=\int_{0}^{t} \Lambda_{s}^{2} d\langle M\rangle_{s}$ is finite, that is, $K_{T}$ is finite $\mathbb{P}$-a.s.

Proposition 2.3 S satisfies the SC condition under Assumption 2.1

Proof. It suffices to show item (a) only. Note that we have

$$
\begin{aligned}
& \left\|[M]_{T}^{1 / 2}+\int_{0}^{T}\left|d A_{t}\right|\right\|_{L^{2}(\mathbb{P})}^{2} \\
& \leq 2 \mathbb{E}\left[[M]_{T}+\left(\int_{0}^{T}\left|d A_{t}\right|\right)^{2}\right] \\
& \leq 2 \mathbb{E}\left[\int_{0}^{T} S_{t-}^{2} \sigma_{t}^{2} d t+\left(\int_{0}^{T} S_{t-}\left|\mu+\left(\beta+\frac{1}{2}\right) \sigma_{t}^{2}\right| d t\right)^{2}\right] \\
& \leq 2 \mathbb{E}\left[\sup _{0 \leq s \leq T} S_{s}^{2}\left\{\int_{0}^{T} \sigma_{t}^{2} d t+\left(|\mu| T+\left|\beta+\frac{1}{2}\right| \int_{0}^{T} \sigma_{t}^{2} d t\right)^{2}\right\}\right] .
\end{aligned}
$$

If $\sup _{0 \leq s \leq T} S_{s} \in L^{2 a}(\mathbb{P})$ holds for a sufficiently small $a>1$, item (a) holds by the Hölder inequality and Lemma 2.4 below.

Now, we take an $a>1$ such that

$$
\left\{2\left(a \beta+\frac{a^{2}}{2}\right)^{+}+a^{2}\right\} \mathcal{B}(T)<\kappa .
$$

Note that we can find such an $a>1$ from the view of (A2) in Assumption 2.1 We shall see $\sup _{0 \leq s \leq T} S_{s} \in L^{2 a}(\mathbb{P})$. Since we have

$$
\begin{aligned}
\int_{0}^{t} \sigma_{s}^{2} d s & =\sigma_{0}^{2} \int_{0}^{t} e^{-\lambda s} d s+\int_{0}^{t} \int_{0}^{s} e^{-\lambda(s-u)} d J_{u} d s \\
& =\sigma_{0}^{2} \mathcal{B}(t)+\int_{0}^{t} \int_{u}^{t} e^{-\lambda(s-u)} d s d J_{u}=\sigma_{0}^{2} \mathcal{B}(t)+\int_{0}^{t} \mathcal{B}(t-u) d J_{u} \\
& \leq \sigma_{0}^{2} \mathcal{B}(t)+\mathcal{B}(t) J_{t} \leq \sigma_{0}^{2} \mathcal{B}(T)+\mathcal{B}(T) J_{t}
\end{aligned}
$$

for any $t \in[0, T]$, we obtain

$$
\begin{aligned}
e^{a L_{t}} & =\exp \left\{a \mu t+a \beta \int_{0}^{t} \sigma_{s}^{2} d s+a \int_{0}^{t} \sigma_{s} d W_{s}\right\} \\
& =\exp \left\{a \mu t-\frac{a^{2}}{2} \int_{0}^{t} \sigma_{s}^{2} d s+a \int_{0}^{t} \sigma_{s} d W_{s}+\left(a \beta+\frac{a^{2}}{2}\right) \int_{0}^{t} \sigma_{s}^{2} d s\right\}
\end{aligned}
$$




$$
\begin{aligned}
& \leq C \exp \left\{-\frac{a^{2}}{2} \int_{0}^{t} \sigma_{s}^{2} d s+a \int_{0}^{t} \sigma_{s} d W_{s}\right. \\
& \left.\quad+\int_{0}^{t} \int_{0}^{\infty} b x \widetilde{N}(d s, d x)+\int_{0}^{t} \int_{0}^{\infty}\left[b x+1-e^{b x}\right] \nu(d x) d s\right\} \\
& =: C Y_{t}^{a, b},
\end{aligned}
$$

where $b:=\left(a \beta+\frac{a^{2}}{2}\right)^{+} \mathcal{B}(T)$, and $C:=\exp \left\{a|\mu| T+b \sigma_{0}^{2}+\int_{0}^{T} \int_{0}^{\infty}\left(e^{b x}-\right.\right.$ 1) $v(d x) d t\}$. Taking into account of (2.3) and (A2) in Assumption 2.1. Lemma 2.5 below yields that $Y^{a, b}$ is a square integrable martingale. Thus, Doob's inequality yields

$$
\begin{aligned}
\mathbb{E}\left[\sup _{0 \leq s \leq T} S_{s}^{2 a}\right] & =\mathbb{E}\left[S_{0}^{2 a} \sup _{0 \leq s \leq T} e^{2 a L_{s}}\right] \leq S_{0}^{2 a} C^{2} \mathbb{E}\left[\sup _{0 \leq s \leq T}\left(Y_{s}^{a, b}\right)^{2}\right] \\
& \leq 4 S_{0}^{2 a} C^{2} \mathbb{E}\left[\left(Y_{T}^{a, b}\right)^{2}\right]<\infty
\end{aligned}
$$

Lemma 2.4 $\int_{0}^{T} \sigma_{t}^{2} d t \in L^{n}(\mathbb{P})$ for any $n \geq 1$.

Proof. From the view of (2.4), it suffices to show $J_{T} \in L^{n}(\mathbb{P})$ for any $n \geq 1$. By Remark 2.2, we have $\mathbb{E}\left[\exp \left\{2 \kappa J_{T}\right\}\right]<\infty$, from which $J_{T} \in L^{n}(\mathbb{P})$ follows for any $n \geq 1$.

Lemma 2.5 For $a \in \mathbb{R}$ and $b \geq 0$, we denote

$$
\begin{aligned}
Y_{t}^{a, b}:= & \exp \left\{-\frac{a^{2}}{2} \int_{0}^{t} \sigma_{s}^{2} d s+a \int_{0}^{t} \sigma_{s} d W_{s}+\int_{0}^{t} \int_{0}^{\infty} b x \widetilde{N}(d s, d x)\right. \\
& \left.+\int_{0}^{t} \int_{0}^{\infty}\left[b x+1-e^{b x}\right] v(d x) d s\right\} .
\end{aligned}
$$

1. If $a$ and $b$ satisfy

$$
\int_{1}^{\infty} \exp \left\{\left(2 b+\frac{a^{2}}{2} \mathcal{B}(T)\right) x\right\} v(d x)<\infty,
$$

then the process $Y^{a, b}$ is a martingale.

2. When we strengthen (2.5) to

$$
\int_{1}^{\infty} \exp \left\{\left(4 b+2 a^{2} \mathcal{B}(T)\right) x\right\} v(d x)<\infty,
$$

$Y^{a, b}$ is a square integrable martingale. 
Proof. 1. From the view of Theorem 1.4 of Ishikawa [5], we need only to show that

(1) $\int_{0}^{\infty}\left[b^{2} x^{2}+\left(1-e^{b x}\right)^{2}\right] v(d x)<\infty$,

(2) $\int_{0}^{\infty}\left[e^{b x} \cdot b x+1-e^{b x}\right] v(d x)<\infty$, and

(3) $\mathbb{E}\left[\exp \left\{\frac{a^{2}}{2} \int_{0}^{T} \sigma_{t}^{2} d t\right\}\right]<\infty$.

By (2.2) and (2.5), conditions (1) and (2) are satisfied. Next, (2.5) and Proposition 3.14 in [4] imply $\mathbb{E}\left[\exp \left\{\frac{a^{2}}{2} \mathcal{B}(T) J_{T}\right\}\right]<\infty$, from which condition (3) follows by (2.4).

2. Denoting $\gamma:=2 b+a^{2} \mathcal{B}(T)$, we have

$$
\begin{aligned}
\left(Y_{T}^{a, b}\right)^{2}=\exp \left\{-a^{2} \int_{0}^{T} \sigma_{s}^{2} d s+2 a \int_{0}^{T} \sigma_{t} d W_{t}\right. \\
\left.+\int_{0}^{T} \int_{0}^{\infty} 2 b x \widetilde{N}(d x, d t)+\int_{0}^{T} \int_{0}^{\infty} 2\left[b x+1-e^{b x}\right] v(d x) d t\right\} \\
\leq \exp \left\{-2 a^{2} \int_{0}^{T} \sigma_{s}^{2} d s+2 a \int_{0}^{T} \sigma_{t} d W_{t}+a^{2} \sigma_{0}^{2} \mathcal{B}(T)+a^{2} \mathcal{B}(T) J_{T}\right. \\
\left.+\int_{0}^{T} \int_{0}^{\infty} 2 b x \widetilde{N}(d x, d t)+\int_{0}^{T} \int_{0}^{\infty} 2\left[b x+1-e^{b x}\right] v(d x) d t\right\} \\
=\exp \left\{-2 a^{2} \int_{0}^{T} \sigma_{s}^{2} d s+2 a \int_{0}^{T} \sigma_{t} d W_{t}+\int_{0}^{T} \int_{0}^{\infty} \gamma x \widetilde{N}(d x, d t)\right. \\
\left.+\int_{0}^{T} \int_{0}^{\infty}\left[\gamma x+2-2 e^{b x}\right] v(d x) d t+a^{2} \sigma_{0}^{2} \mathcal{B}(T)\right\} \\
=\exp \left\{\int_{0}^{T} \int_{0}^{\infty}\left[1-2 e^{b x}+e^{\gamma x}\right] v(d x) d t+a^{2} \sigma_{0}^{2} \mathcal{B}(T)\right\} Y_{T}^{2 a, \gamma} .
\end{aligned}
$$

Under (2.6), we have $\int_{1}^{\infty} \exp \{2 \gamma x\} v(d x)<\infty$. Thus, we can see that $\gamma^{2 a, \gamma}$ is a martingale by the same sort argument as item 1. Moreover, we have $\int_{0}^{\infty}\left[1-2 e^{b x}+e^{\gamma x}\right] v(d x)<\infty$, from which the square integrability of $Y_{T}^{a, b}$ follows.

Next, we give a definition of LRM based on Theorem 1.6 of Schweizer [11].

Definition 2.6 1. $\Theta_{S}$ denotes the space of all $\mathbb{R}$-valued predictable processes $\xi$ satisfying $\mathbb{E}\left[\int_{0}^{T} \xi_{t}^{2} d\langle M\rangle_{t}+\left(\int_{0}^{T}\left|\xi_{t} d A_{t}\right|\right)^{2}\right]<\infty$.

2. An $L^{2}$-strategy is given by a pair $\varphi=(\xi, \eta)$, where $\xi \in \Theta_{S}$ and $\eta$ is an adapted process such that $V(\varphi):=\xi S+\eta$ is a right continuous process with $\mathbb{E}\left[V_{t}^{2}(\varphi)\right]<\infty$ for every $t \in[0, T]$. Note that $\xi_{t}$ (resp. $\eta_{t}$ ) represents the amount of units of the risky asset (resp. the risk-free asset) an investor holds at time $t$.

3. For claim $F \in L^{2}(\mathbb{P})$, the process $C^{F}(\varphi)$ defined by $C_{t}^{F}(\varphi):=F 1_{\{t=T\}}+$ $V_{t}(\varphi)-\int_{0}^{t} \xi_{s} d S_{s}$ is called the cost process of $\varphi=(\xi, \eta)$ for $F$. 
4. An $L^{2}$-strategy $\varphi$ is said local risk-minimization (LRM) for claim $F$ if $V_{T}(\varphi)=$ 0 and $C^{F}(\varphi)$ is a martingale orthogonal to $M$, that is, $\left[C^{F}(\varphi), M\right]$ is a uniformly integrable martingale.

5. An $F \in L^{2}(\mathbb{P})$ admits a Föllmer-Schweizer decomposition (FS decomposition, for short) if it can be described by

$$
F=F_{0}+\int_{0}^{T} \xi_{t}^{F} d S_{t}+L_{T}^{F}
$$

where $F_{0} \in \mathbb{R}, \xi^{F} \in \Theta_{S}$ and $L^{F}$ is a square-integrable martingale orthogonal to $M$ with $L_{0}^{F}=0$.

For more details on LRM, see Schweizer [10], [11]. Now, we introduce a relationship between LRM and FS decomposition.

Proposition 2.7 Under Assumption 2.1. $L R M \varphi=(\xi, \eta)$ for F exists if and only if $F$ admits an FS decomposition; and its relationship is given by

$$
\xi_{t}=\xi_{t}^{F}, \quad \eta_{t}=F_{0}+\int_{0}^{t} \xi_{s}^{F} d S_{s}+L_{t}^{F}-F 1_{\{t=T\}}-\xi_{t}^{F} S_{t} .
$$

Proof. This is from Proposition 5.2 of [11], together with Proposition 2.3.

Thus, it suffices to get a representation of $\xi^{F}$ in (2.7) in order to obtain LRM for claim $F$. Henceforth, we identify $\xi^{F}$ with LRM for $F$.

\subsection{Minimal martingale measure}

We need to study upon the MMM in order to discuss FS decomposition. A probability measure $\mathbb{P}^{*} \sim \mathbb{P}$ is called the minimal martingale measure (MMM), if $S$ is a $\mathbb{P}^{*}$-martingale; and any square-integrable $\mathbb{P}$-martingale orthogonal to $M$ remains a martingale under $\mathbb{P}^{*}$. Now, we consider the following SDE:

$$
d Z_{t}=-Z_{t-} \Lambda_{t} d M_{t}, \quad Z_{0}=1 .
$$

The solution to (2.8) is a stochastic exponential of $-\int_{0}^{\cdot} \Lambda_{t} d M_{t}$. More precisely, denoting

$$
u_{t}:=\Lambda_{t} S_{t-} \sigma_{t}=\frac{\mu}{\sigma_{t}}+\left(\beta+\frac{1}{2}\right) \sigma_{t}
$$

for $t \in[0, T]$, we have $\Lambda_{t} d M_{t}=u_{t} d W_{t}$; and

$$
Z_{t}=\exp \left\{-\frac{1}{2} \int_{0}^{t} u_{s}^{2} d s-\int_{0}^{t} u_{s} d W_{s}\right\} .
$$

To see that $Z_{T}$ becomes the density of the MMM, it is enough to show the square integrability of $Z_{T}$. 
Proposition 2.8 $Z_{T} \in L^{2}(\mathbb{P})$.

Proof. First of all, there is a constant $C_{u}>0$ such that

$$
u_{t}^{2}=\frac{\mu^{2}}{\sigma_{t}^{2}}+2 \mu\left(\beta+\frac{1}{2}\right)+\left(\beta+\frac{1}{2}\right)^{2} \sigma_{t}^{2} \leq C_{u}+\left(\beta+\frac{1}{2}\right)^{2} \sigma_{t}^{2}
$$

by (2.9). Thus, (2.10) implies

$$
\begin{aligned}
Z_{T}^{2}= & \exp \left\{-2 \int_{0}^{T} u_{t}^{2} d t-\int_{0}^{T} 2 u_{t} d W_{t}+\int_{0}^{T} u_{t}^{2} d t\right\} \\
\leq & \exp \left\{-2 \int_{0}^{T} u_{t}^{2} d t-\int_{0}^{T} 2 u_{t} d W_{t}+T C_{u}+\left(\beta+\frac{1}{2}\right)^{2} \int_{0}^{T} \sigma_{t}^{2} d t\right\} \\
\leq & \exp \left\{-2 \int_{0}^{T} u_{t}^{2} d t-\int_{0}^{T} 2 u_{t} d W_{t}+T C_{u}+\left(\beta+\frac{1}{2}\right)^{2}\left[\sigma_{0}^{2} \mathcal{B}(T)+\mathcal{B}(T) J_{T}\right]\right\} \\
\leq & \exp \left\{T C_{u}+\left(\beta+\frac{1}{2}\right)^{2} \sigma_{0}^{2} \mathcal{B}(T)+\int_{0}^{T} \int_{0}^{\infty}\left[e^{\kappa x}-1\right] v(d x) d t\right\} \\
& \times \exp \left\{-2 \int_{0}^{T} u_{t}^{2} d t-\int_{0}^{T} 2 u_{t} d W_{t}+\int_{0}^{T} \int_{0}^{\infty} \kappa x \widetilde{N}(d x, d t)\right. \\
& \left.+\int_{0}^{T} \int_{0}^{\infty}\left[\kappa x+1-e^{\kappa x}\right] v(d x) d t\right\},
\end{aligned}
$$

since $\left(\beta+\frac{1}{2}\right)^{2} \mathcal{B}(T) \leq \kappa$ by (A2). In addition, Remark2.2 implies

$$
\begin{aligned}
\mathbb{E}\left[\exp \left\{2 \int_{0}^{T} u_{t}^{2} d t\right\}\right] & \leq \mathbb{E}\left[\exp \left\{2 T C_{u}+2\left(\beta+\frac{1}{2}\right)^{2} \int_{0}^{T} \sigma_{t}^{2} d t\right\}\right] \\
& \leq \exp \left\{2 T C_{u}+2 \kappa \sigma_{0}^{2}\right\} \mathbb{E}\left[e^{2 \kappa J_{T}}\right]<\infty .
\end{aligned}
$$

Hence, we can see that $Z_{T}^{2}$ is integrable by the same manner as the proof of item 1 in Lemma 2.5 .

Henceforth, we denote the MMM by $\mathbb{P}^{*}$, that is, we have $Z_{T}=\frac{d \mathbb{P}^{*}}{d \mathbb{P}}$. Note that $d W_{t}^{\mathbb{P}^{*}}:=d W_{t}+u_{t} d t$ is a Brownian motion under $\mathbb{P}^{*}$; and $\widetilde{N}$ remains a martingale under $\mathbb{P}^{*}$. Remark that we can rewrite (2.1) and $L_{T}$ as $d S_{t}=S_{t-} \sigma_{t} d W_{t}^{\mathbb{P}^{*}}$ and $L_{T}=\int_{0}^{T} \sigma_{s} d W_{s}^{\mathbb{P}^{*}}-\frac{1}{2} \int_{0}^{T} \sigma_{s}^{2} d s$, respectively. The following lemma is indispensable to formulate a Malliavin calculus under $\mathbb{P}^{*}$.

Lemma 2.9 $W^{\mathbb{P}^{*}}$ is independent of $\widetilde{N}$; and $W_{t}^{\mathbb{P}^{*}}+\int_{0}^{t} \int_{0}^{\infty} z \widetilde{N}(d s, d z)\left(=: X_{t}^{*}\right)$ is a Léry process under $\mathbb{P}^{*}$.

Proof. This is given from Theorem 3.2 in [6]. Remark that Assumptions Z1 $\mathrm{Z3}$ in [6] are their standing assumptions. Assumptions Z1 and Z2 are satisfied 
in our setting from Assumption 2.1. On the other hand, Assumption Z3 does not necessarily hold, but it is not needed for Theorem 3.2 in [6].

Remark 2.10 The filtration $\mathbb{F}$ coincides with the augmented filtration generated by $W^{\mathbb{P}^{*}}$ and $\widetilde{N}$.

\subsection{Malliavin calculus under $\mathbb{P}^{*}$}

Here, regarding $\left(\Omega, \mathcal{F}, \mathbb{P}^{*}\right)$ as the underlying probability space, we formulate a Malliavin calculus for $X^{*}$ under $\mathbb{P}^{*}$ based on Petrou [7] and Chapter 5 of Renauld [8]. Although [1] adopted the canonical Lévy space framework undertaken by Solé et al. [12], we need to take a different way to define a Malliavin derivative, since the property of the canonical Lévy space is not preserved under change of measure.

First of all, we need to prepare some notation; and define iterated integrals with respect to $W^{\mathbb{P}^{*}}$ and $\widetilde{N}$. Denoting $U_{0}:=[0, T]$ and $U_{1}:=[0, T] \times(0, \infty)$, we define

$$
\begin{aligned}
Q_{0}(A) & :=\int_{A} d W_{t}^{\mathbb{P}^{*}} \quad \text { for any } A \in \mathcal{B}\left(U_{0}\right), \\
Q_{1}(A) & :=\int_{A} \widetilde{N}(d t, d x) \quad \text { for any } A \in \mathcal{B}\left(U_{1}\right), \\
\left\langle Q_{0}\right\rangle & :=m, \quad \text { and } \quad\left\langle Q_{1}\right\rangle:=m \times v,
\end{aligned}
$$

where $m$ is the Lebesgue measure on $U_{0}$. We denote

$$
G_{\left(j_{1}, \ldots, j_{n}\right)}:=\left\{\left(u_{1}^{j_{1}}, \ldots, u_{n}^{j_{n}}\right) \in \prod_{k=1}^{n} U_{j_{k}}: 0<t_{1}<\cdots<t_{n}<T\right\}
$$

for $n \in \mathbb{N}$ and $\left(j_{1}, \ldots, j_{n}\right) \in\{0,1\}^{n}$, where $u_{k}^{j_{k}}:=t_{k}$ if $j_{k}=0$; and $:=\left(t_{k}, x\right)$ if $j_{k}=1$ for $k=1, \ldots, n$. We define an $n$-fold iterated integral as follows:

$$
J_{n}^{\left(j_{1}, \ldots, j_{n}\right)}\left(g_{n}^{\left(j_{1}, \ldots, j_{n}\right)}\right):=\int_{G_{\left(j_{1}, \ldots, j_{n}\right)}} g_{n}^{\left(j_{1}, \ldots, j_{n}\right)}\left(u_{1}^{j_{1}}, \ldots, u_{n}^{j_{n}}\right) Q_{j_{1}}\left(d u_{1}^{j_{1}}\right) \cdots Q_{j_{n}}\left(d u_{n}^{j_{n}}\right),
$$

where $g_{n}^{\left(j_{1}, \ldots, j_{n}\right)}$ is a deterministic function in $L^{2}\left(G_{\left(j_{1}, \ldots, j_{n}\right)}, \bigotimes_{k=1}^{n}\left\langle Q_{j_{k}}\right\rangle\right)$. Then, Theorem 1 in [7] ensures that every $L^{2}\left(\mathbb{P}^{*}\right)$ random variable $F$ is represented as a sum of iterated integrals, that is, we can find deterministic functions $g_{n}^{\left(j_{1}, \ldots, j_{n}\right)} \in L^{2}\left(G_{\left(j_{1}, \ldots, j_{n}\right)}, \bigotimes_{k=1}^{n}\left\langle Q_{j_{k}}\right\rangle\right)$ for $n \in \mathbb{N}$ and $\left(j_{1}, \ldots, j_{n}\right) \in\{0,1\}^{n}$ such that $F$ has the following chaos expansion:

$$
F=\mathbb{E}_{\mathbb{P}^{*}}[F]+\sum_{n=1}^{\infty} \sum_{\left(j_{1}, \ldots, j_{n}\right) \in\{0,1\}^{n}} J_{n}^{\left(j_{1}, \ldots, j_{n}\right)}\left(g_{n}^{\left(j_{1}, \ldots, j_{n}\right)}\right) .
$$

Note that the infinite series in (2.11) converges in $L^{2}\left(\mathbb{P}^{*}\right)$. 
Now, we define $\mathbb{D}^{0}$ the space of Malliavin differentiable random variables; and a Malliavin derivative operator $D^{0}$. Denoting, for $1 \leq k \leq n$ and $t \in(0, T)$,

$$
\begin{aligned}
G_{\left(j_{1}, \ldots, j_{n}\right)}^{k}(t):= & \left\{\left(u_{1}^{j_{1}}, \ldots, u_{k-1}^{j_{k-1}}, u_{k+1}^{j_{k+1}} \ldots, u_{n}^{j_{n}}\right) \in G_{\left(j_{1}, \ldots, j_{k-1}, j_{k+1}, \ldots, j_{n}\right)}:\right. \\
& \left.0<t_{1}<\cdots<t_{k-1}<t<t_{k+1}<\cdots<t_{n}<T\right\},
\end{aligned}
$$

we define $\mathbb{D}^{0}$ as

$$
\begin{aligned}
\mathbb{D}^{0}:=\{ & F \in L^{2}\left(\mathbb{P}^{*}\right), F=\mathbb{E}_{\mathbb{P}^{*}}[F]+\sum_{n=1}^{\infty} \sum_{\left(j_{1}, \ldots, j_{n}\right) \in\{0,1\}^{n}} J_{n}^{\left(j_{1}, \ldots, j_{n}\right)}\left(g_{n}^{\left(j_{1}, \ldots, j_{n}\right)}\right): \\
& \left\|g_{1}^{(0)}\right\|_{L^{2}(m)}^{2}+\sum_{n=2}^{\infty} \sum_{\left(j_{1}, \ldots, j_{n}\right) \in\{0,1\}^{n}} \sum_{k=1}^{n} \mathbf{1}_{\left\{j_{k}=0\right\}} \\
& \left.\times \int_{0}^{T}\left\|g_{n}^{\left(j_{1}, \ldots, j_{k-1}, 0, j_{k+1,}, \ldots, j_{n}\right)}(\ldots, t, \ldots)\right\|_{L^{2}\left(G_{\left(j_{1}, \ldots, j_{n}\right)}^{k}(t)\right)}^{2} d t<\infty\right\} .
\end{aligned}
$$

Moreover, for $F \in \mathbb{D}^{0}$ and $t \in[0, T]$, we define

$$
\begin{aligned}
D_{t}^{0} F:= & g_{1}^{(0)}(t)+\sum_{n=2}^{\infty} \sum_{\left(j_{1}, \ldots, j_{n}\right) \in\{0,1\}^{n}} \sum_{k=1}^{n} \mathbf{1}_{\left\{j_{k}=0\right\}} \\
& \times J_{n-1}^{\left(j_{1}, \ldots, j_{k-1}, j_{k+1}, \ldots, j_{n}\right)}\left(g_{n}^{\left(j_{1}, \ldots, j_{k-1}, 0, j_{k+1}, \ldots, j_{n}\right)}(\ldots, t, \ldots) \mathbf{1}_{G_{\left(j_{1}, \ldots, j n\right.}^{k}(t)}\right) .
\end{aligned}
$$

\section{Main results}

We give explicit representations of LRM for call and put options as our main results. As in [1], we consider firstly put options, since a Malliavin derivative for put options is given owing to its boundedness. LRM for call options will be given as a corollary. If we dealt with call options firstly, then we would need to impose additional assumptions.

Before stating our main theorem, we prepare two propositions, one is a Malliavin derivative for put options; and the other is a Clark-Ocone type representation result for random variables in $\mathbb{D}^{0}$.

Proposition 3.1 For $K>0$, we have $\left(K-S_{T}\right)^{+} \in \mathbb{D}^{0}$, and

$$
D_{t}^{0}\left(K-S_{T}\right)^{+}=-\mathbf{1}_{\left\{S_{T}<K\right\}} S_{T} \sigma_{t} .
$$

Proof. The same result has been given in Proposition 4.1 of [1]. However, their framework of Malliavin calculus is different from ours as said at the beginning of Subsection 2.3. Thus, we give a proof anew by the same way as [1].

First of all, by the same argument as Lemma A.1 in [1], we have $D_{t}^{0} \sigma_{s}^{2}=0$. Theorem 2 in [7] implies $D_{t}^{0} \sigma_{s}=0$ by the same manner as Lemma A.2 of [1]. In addition, by the same way as Lemmas A.3 and A.4 in [1], we can see that 
$D_{t}^{0} \int_{0}^{T} \sigma_{s}^{2} d s=0$; and $D_{t}^{0} \int_{0}^{T} \sigma_{s} d W_{s}^{\mathbb{P}^{*}}=\sigma_{t}$ by using Proposition 6 in [7]. As a result, we obtain $L_{T} \in \mathbb{D}^{0}$ and $D_{t}^{0} L_{T}=\sigma_{t}$. Next, denoting

$$
f_{K}(r):= \begin{cases}S_{0} e^{r}, & \text { if } r \leq \log \left(K / S_{0}\right), \\ K r+K\left(1-\log \left(K / S_{0}\right)\right), & \text { if } r>\log \left(K / S_{0}\right) .\end{cases}
$$

we have that $f_{K} \in C^{1}(\mathbb{R})$ and $0<f_{K}^{\prime}(r) \leq K$ for any $r \in \mathbb{R}$. Thus, Theorem 2 of [7] implies that $f_{K}\left(L_{T}\right) \in \mathbb{D}^{0}$ and

$$
D_{t}^{0} f_{K}\left(L_{T}\right)=f_{K}^{\prime}\left(L_{T}\right) D_{t}^{0} L_{T}=f_{K}^{\prime}\left(L_{T}\right) \sigma_{t} .
$$

Since $\left(K-S_{T}\right)^{+}=\left(K-f_{K}\left(L_{T}\right)\right)^{+}$, we need only to see $\left(K-f_{K}\left(L_{T}\right)\right)^{+} \in$ $\mathbb{D}^{0}$; and calculate $D_{t}^{0}\left(K-f_{K}\left(L_{T}\right)\right)^{+}$. To this end, we take a mollifier function $\varphi$ which is a $C^{\infty}$-function from $\mathbb{R}$ to $[0, \infty)$ with $\operatorname{supp}(\varphi) \subset[-1,1]$ and $\int_{-\infty}^{\infty} \varphi(x) d x=1$. We denote $\varphi_{n}(x):=n \varphi(n x)$ and $g_{n}(x):=\int_{-\infty}^{\infty}(K-$ $y)^{+} \varphi_{n}(x-y) d y$ for any $n \geq 1$. Noting that

$$
g_{n}(x)=\int_{-\infty}^{\infty}\left(K-x+\frac{y}{n}\right)^{+} \varphi(y) d y=\int_{-n(K-x)}^{\infty}\left(K-x+\frac{y}{n}\right) \varphi(y) d y,
$$

we have $g_{n}^{\prime}(x)=-\int_{-n(K-x)}^{\infty} \varphi(y) d y$, so that $g_{n} \in C^{1}$ and $\left|g_{n}^{\prime}\right| \leq 1$. Thus, Theorem 2 in [7] again implies that, for any $n \geq 1, g_{n}\left(f_{K}\left(L_{T}\right)\right) \in \mathbb{D}^{0}$ and

$$
D_{t}^{0} g_{n}\left(f_{K}\left(L_{T}\right)\right)=g_{n}^{\prime}\left(f_{K}\left(L_{T}\right)\right) D_{t}^{0} f_{K}\left(L_{T}\right)=g_{n}^{\prime}\left(f_{K}\left(L_{T}\right)\right) f_{K}^{\prime}\left(L_{T}\right) \sigma_{t}
$$

by (3.1). We have then

$$
\sup _{n \geq 1}\left\|D^{0} g_{n}\left(f_{K}\left(L_{T}\right)\right)\right\|_{L^{2}\left(m \times \mathbb{P}^{*}\right)}^{2} \leq K^{2} \mathbb{E}_{\mathbb{P}^{*}}\left[\int_{0}^{T} \sigma_{t}^{2} d t\right]<\infty .
$$

In addition, noting that

$$
\begin{aligned}
\left|g_{n}(x)-(K-x)^{+}\right| & =\left|\int_{-1}^{1}\left\{\left(K-x+\frac{y}{n}\right)^{+}-(K-x)^{+}\right\} \varphi(y) d y\right| \\
& \leq \frac{1}{n} \int_{-1}^{1}|y| \varphi(y) d y \leq \frac{1}{n}
\end{aligned}
$$

for any $x \in \mathbb{R}$, we have $\lim _{n \rightarrow \infty} \mathbb{E}\left[\left|g_{n}\left(f_{K}\left(L_{T}\right)\right)-\left(K-f_{K}\left(L_{T}\right)\right)^{+}\right|^{2}\right]=0$. As a result, Lemma 3.2 below implies that $\left(K-f_{K}\left(L_{T}\right)\right)^{+} \in \mathbb{D}^{0}$. Furthermore, Lemma 2 of [7] ensures the existence of a subsequence $n_{k}$ such that $D^{0} g_{n_{k}}\left(f_{K}\left(L_{T}\right)\right)$ converges to $D^{0}\left(K-f_{K}\left(L_{T}\right)\right)^{+}$in the sense of $L^{2}\left(m \times \mathbb{P}^{*}\right)$. On the other hand, we have $\lim _{n \rightarrow \infty} g_{n}^{\prime}(x)=-\mathbf{1}_{\{x<K\}}-\mathbf{1}_{\{x=K\}} \int_{0}^{\infty} \varphi(y) d y$; and $\mathbb{P}^{*}\left(f_{K}\left(L_{T}\right)=K\right)=0$ by Corollary 2.3 of [6], from which $\lim _{n \rightarrow \infty} g_{n}^{\prime}\left(f_{K}\left(L_{T}\right)\right)=-\mathbf{1}_{\left\{f_{K}\left(L_{T}\right)<K\right\}}$ a.s. follows. Consequently, by taking a further subsequence if need be, (3.2) provides

$$
D_{t}^{0}\left(K-S_{T}\right)^{+}=D_{t}^{0}\left(K-f_{K}\left(L_{T}\right)\right)^{+}=\lim _{k \rightarrow \infty} D_{t}^{0} g_{n_{k}}\left(f_{K}\left(L_{T}\right)\right)
$$




$$
\begin{aligned}
& =\lim _{k \rightarrow \infty} g_{n_{k}}^{\prime}\left(f_{K}\left(L_{T}\right)\right) f_{K}^{\prime}\left(L_{T}\right) \sigma_{t}=-\mathbf{1}_{\left\{f_{K}\left(L_{T}\right)<K\right\}} f_{K}^{\prime}\left(L_{T}\right) \sigma_{t} \\
& =-\mathbf{1}_{\left\{S_{T}<K\right\}} S_{T} \sigma_{t}, \quad m \times \mathbb{P}^{*} \text {-a.s. }
\end{aligned}
$$

Lemma 3.2 Let $F$ be in $L^{2}\left(\mathbb{P}^{*}\right)$, and $\left(F_{n}\right)_{n>1}$ a sequence of $\mathbb{D}^{0}$ converging to $F$ in $L^{2}\left(\mathbb{P}^{*}\right)$. If $\sup _{n \geq 1}\left\|D^{0} F_{n}\right\|_{L^{2}\left(m \times \mathbb{P}^{*}\right)}<\infty$, then $F \in \mathbb{D}^{0}$.

Proof. This is given from the proof of Lemma 5.5.5 of [8].

Proposition 3.3 For $F \in \mathbb{D}^{0}$, we have

$$
F=\mathbb{E}_{\mathbb{P}^{*}}[F]+\int_{0}^{T} \mathbb{E}_{\mathbb{P}^{*}}\left[D_{t}^{0} F \mid \mathcal{F}_{t-}\right] d W_{t}^{\mathbb{P}^{*}}+\int_{0}^{T} \int_{0}^{\infty} \psi_{t, x} \tilde{N}(d t, d x)
$$

for some predictable process $\psi \in L^{2}\left(m \times v \times \mathbb{P}^{*}\right)$.

Proof. Denoting by (2.11) the chaos expansion of $F$, we have

$$
\begin{aligned}
& F=\mathbb{E}_{\mathbb{P}^{*}}[F]+\sum_{n=1}^{\infty} \sum_{\left(j_{1}, \ldots, j_{n-1}\right) \in\{0,1\}^{n-1}}\left\{J_{n}^{\left(j_{1}, \ldots, j_{n-1}, 0\right)}\left(g_{n}^{\left(j_{1}, \ldots, j_{n-1}, 0\right)}\right)+J_{n}^{\left(j_{1}, \ldots, j_{n-1}, 1\right)}\left(g_{n}^{\left(j_{1}, \ldots, j_{n-1}, 1\right)}\right)\right\} \\
& =\mathbb{E}_{\mathbb{P}^{*}}[F]+\int_{0}^{T} g_{1}^{(0)}(t) d W_{t}^{\mathbb{P}^{*}}+\sum_{n=2}^{\infty} \sum_{\left(j_{1}, \ldots, j_{n-1}\right) \in\{0,1\}^{n-1}} \int_{0}^{T} J_{n-1}^{\left(j_{1}, \ldots, j_{n-1}\right)}\left(g_{n}^{\left(j_{1}, \ldots, j_{n-1}, 0\right)}(\ldots, t) \mathbf{1}_{G_{\left(j_{1}, \ldots, j n\right)}^{n}(t)}\right) d W_{t}^{\mathbb{P}^{*}} \\
& +\int_{0}^{T} \int_{0}^{\infty} g_{1}^{(1)}((t, x)) \widetilde{N}(d t, d x) \\
& +\sum_{n=2}^{\infty} \sum_{\left(j_{1}, \ldots, j_{n-1}\right) \in\{0,1\}^{n-1}} \int_{0}^{T} \int_{0}^{\infty} J_{n-1}^{\left(j_{1}, \ldots, j_{n-1}\right)}\left(g_{n}^{\left(j_{1}, \ldots, j_{n-1}, 1\right)}(\ldots,(t, x)) \mathbf{1}_{\left.G_{\left(j_{1}, \ldots, j n\right.}^{n}\right)}(t)\right) \widetilde{N}(d t, d x) \\
& =\mathbb{E}_{\mathbb{P}^{*}}[F]+\int_{0}^{T}\left\{g_{1}^{(0)}(t)+\sum_{n=2}^{\infty} \sum_{\left(j_{1}, \ldots, j_{n-1}\right) \in\{0,1\}^{n-1}} J_{n-1}^{\left(j_{1}, \ldots, j_{n-1}\right)}\left(g_{n}^{\left(j_{1}, \ldots, j_{n-1}, 0\right)}(\ldots, t) \mathbf{1}_{\left.G_{\left(j_{1}, \ldots, j n\right.}^{n}\right)}(t)\right)\right\} d W_{t}^{\mathbb{P}^{*}} \\
& +\int_{0}^{T} \int_{0}^{\infty}\left\{g_{1}^{(1)}((t, x))+\sum_{n=2}^{\infty} \sum_{\left(j_{1}, \ldots, j_{n-1}\right) \in\{0,1\}^{n-1}} J_{n-1}^{\left(j_{1}, \ldots, j_{n-1}\right)}\left(g_{n}^{\left(j_{1}, \ldots, j_{n-1,1}\right)}(\ldots,(t, x)) \mathbf{1}_{G_{\left(j_{1}, \ldots, j n\right)}}(t)\right)\right\} \widetilde{N}(d t, d x) \\
& =: \mathbb{E}_{\mathbb{P}^{*}}[F]+\int_{0}^{T} \phi_{t} d W_{t}^{\mathbb{P}^{*}}+\int_{0}^{T} \int_{0}^{\infty} \psi_{t, x} \widetilde{N}(d t, d x) .
\end{aligned}
$$

The above third equality (3.3) is proved in Lemma 3.4 below. On the other hand, noting that $F \in \mathbb{D}^{0}$, we have

$$
\begin{aligned}
\mathbb{E}_{\mathbb{P}^{*}}\left[D_{t}^{0} F \mid \mathcal{F}_{t-}\right]= & \mathbb{E}_{\mathbb{P}^{*}}\left[g_{1}^{(0)}(t)+\sum_{n=2}^{\infty} \sum_{\left(j_{1}, \ldots, j_{n}\right) \in\{0,1\}^{n}} \sum_{k=1}^{n} \mathbf{1}_{\left\{j_{k}=0\right\}}\right. \\
& \left.\times J_{n-1}^{\left(j_{1}, \ldots, j_{k-1}, j_{k+1}, \ldots, j_{n}\right)}\left(g_{n}^{\left(j_{1}, \ldots, j_{k-1}, 0, j_{k+1}, \ldots, j_{n}\right)}(\ldots, t, \ldots) \mathbf{1}_{G_{\left(j_{1}, \ldots, j n\right)}^{k}(t)}\right) \mid \mathcal{F}_{t-}\right] \\
= & g_{1}^{(0)}(t)+\sum_{n=2}^{\infty} \sum_{\left(j_{1}, \ldots, j_{n}\right) \in\{0,1\}^{n}} \sum_{k=1}^{n} \mathbf{1}_{\left\{j_{k}=0\right\}}
\end{aligned}
$$




$$
\begin{aligned}
& \times \mathbb{E}_{\mathbb{P}^{*}}\left[J_{n-1}^{\left(j_{1}, \ldots, j_{k-1}, j_{k+1}, \ldots, j_{n}\right)}\left(g_{n}^{\left(j_{1}, \ldots, j_{k-1}, 0, j_{k+1}, \ldots, j_{n}\right)}(\ldots, t, \ldots) \mathbf{1}_{G_{\left(j_{1}, \ldots, j n\right)}^{k}(t)}\right) \mid \mathcal{F}_{t-}\right] \\
= & g_{1}^{(0)}(t)+\sum_{n=2}^{\infty} \sum_{\left(j_{1}, \ldots, j_{n}\right) \in\{0,1\}^{n}} \mathbf{1}_{\left\{j_{n}=0\right\}} \int_{n-1}^{\left(j_{1}, \ldots, j_{n-1}\right)}\left(g_{n}^{\left(j_{1}, \ldots, j_{n-1}, 0\right)}(\ldots, t) \mathbf{1}_{G_{\left(j_{1}, \ldots, j n\right)}}(t)\right) \\
= & \phi_{t} .
\end{aligned}
$$

As a result, $\phi$ belongs to $L^{2}\left(m \times \mathbb{P}^{*}\right)$. Thus, $\int_{0}^{T} \int_{0}^{\infty} \psi_{t, x} \tilde{N}(d t, d x)$ is square integrable, that is, $\psi \in L^{2}\left(m \times v \times \mathbb{P}^{*}\right)$. This completes the proof of Proposition 3.3.

Lemma 3.4 (3.3) in the proof of Proposition 3.3 holds true. In other words, we have, for $l=0,1$,

$$
\begin{aligned}
& \sum_{n=2}^{\infty} \sum_{\left(j_{1}, \ldots, j_{n-1}\right) \in\{0,1\}^{n-1}} \int_{U_{l}} J_{n-1}^{\left(j_{1}, \ldots, j_{n-1}\right)}\left(g_{n}^{\left(j_{1}, \ldots, j_{n-1}, l\right)}\left(\ldots, \widehat{u}^{l}\right) \mathbf{1}_{G_{\left(j_{1}, \ldots, j n\right)}^{n}(t)}\right) Q_{l}\left(d \widehat{u}^{l}\right) \\
& =\int_{U_{l}} \sum_{n=2}^{\infty} \sum_{\left(j_{1}, \ldots, j_{n-1}\right) \in\{0,1\}^{n-1}} J_{n-1}^{\left(j_{1}, \ldots, j_{n-1}\right)}\left(g_{n}^{\left(j_{1}, \ldots, j_{n-1}, l\right)}\left(\ldots, \widehat{u}^{l}\right) \mathbf{1}_{\left.G_{\left(j_{1}, \ldots, j n\right.}^{n}\right)}(t)\right) Q_{l}\left(d \widehat{u}^{l}\right),
\end{aligned}
$$

where $\widehat{u}^{0}=t \in U_{0}$ and $\widehat{u}^{1}=(t, x) \in U_{1}$.

Proof. Recall that the infinite series in a chaos expansion converges in the $L^{2}\left(\mathbb{P}^{*}\right)$-sense. Now, for $l=0,1$, we denote

$$
\Phi^{l, N}\left(\widehat{u}^{l}\right):=\sum_{n=2}^{N} \sum_{\left(j_{1}, \ldots, j_{n-1}\right) \in\{0,1\}^{n-1}} J_{n-1}^{\left(j_{1}, \ldots, j_{n-1}\right)}\left(g_{n}^{\left(j_{1}, \ldots, j_{n-1}, l\right)}\left(\ldots, \widehat{u}^{l}\right) \mathbf{1}_{G_{\left(j_{1}, \ldots, j n\right)}^{n}(t)}\right)
$$

for $N \geq 2$, and

$$
\Phi^{l}\left(\widehat{u}^{l}\right):=\sum_{n=2}^{\infty} \sum_{\left(j_{1}, \ldots, j_{n-1}\right) \in\{0,1\}^{n-1}} J_{n-1}^{\left(j_{1}, \ldots, j_{n-1}\right)}\left(g_{n}^{\left(j_{1}, \ldots, j_{n-1}, l\right)}\left(\ldots, \widehat{u}^{l}\right) \mathbf{1}_{G_{\left(j_{1}, \ldots, j n\right)}^{n}(t)}\right) .
$$

We have then that, for $l=0,1,\left(\Phi^{l, N}\right)_{N \geq 2}$ is a sequence of $L^{2}\left(\left\langle Q_{l}\right\rangle \times \mathbb{P}^{*}\right)$ converging to $\Phi^{l}$ in the $L^{2}\left(\left\langle Q_{l}\right\rangle \times \mathbb{P}^{*}\right)$-sense. Thus, we have

$$
\lim _{N \rightarrow \infty} \mathbb{E}_{\mathbb{P}^{*}}\left[\left|\int_{U_{l}} \Phi^{l, N}\left(\widehat{u}^{l}\right) Q_{l}\left(d \widehat{u}^{l}\right)-\int_{U_{l}} \Phi^{l}\left(\widehat{u}^{l}\right) Q_{l}\left(d \widehat{u}^{l}\right)\right|^{2}\right]=0 .
$$

The following theorem is our main result.

Theorem 3.5 For $K>0, L R M \xi^{\left(K-S_{T}\right)^{+}}$of put option $\left(K-S_{T}\right)^{+}$is represented as

$$
\xi_{t}^{\left(K-S_{T}\right)^{+}}=\frac{-1}{S_{t-}} \mathbb{E}_{\mathbb{P}^{*}}\left[\mathbf{1}_{\left\{S_{T}<K\right\}} S_{T} \mid \mathcal{F}_{t-}\right] .
$$


Proof. Denoting by $\zeta_{t}$ the right hand side of (3.4), we shall see that the process $\zeta$ is in $\Theta_{S}$. Noting that $\left|\zeta_{t}\right| \leq \frac{K}{S_{t-}}$, we have

$$
\begin{aligned}
& \mathbb{E}\left[\int_{0}^{T} \zeta_{t}^{2} d\langle M\rangle_{t}+\left(\int_{0}^{T}\left|\zeta_{t} d A_{t}\right|\right)^{2}\right] \\
& \leq \mathbb{E}\left[\int_{0}^{T} K^{2} \sigma_{t}^{2} d t+\left(\int_{0}^{T} K\left|\mu+\left(\beta+\frac{1}{2}\right) \sigma_{t}^{2}\right| d t\right)^{2}\right]<\infty,
\end{aligned}
$$

since $\mathbb{E}\left[\left(\int_{0}^{T} \sigma_{t}^{2} d t\right)^{2}\right]<\infty$ by Lemma 2.4. As a result, $\zeta \in \Theta_{S}$ holds.

Next, defining

$$
L_{t}^{\left(K-S_{T}\right)^{+}}:=\mathbb{E}\left[\left(K-S_{T}\right)^{+}-\mathbb{E}_{\mathbb{P}^{*}}\left[\left(K-S_{T}\right)^{+}\right]-\int_{0}^{T} \zeta_{s} d S_{s} \mid \mathcal{F}_{t}\right],
$$

we show that

$$
\left(K-S_{T}\right)^{+}=\mathbb{E}_{\mathbb{P}^{*}}\left[\left(K-S_{T}\right)^{+}\right]+\int_{0}^{T} \zeta_{t} d S_{t}+L_{T}^{\left(K-S_{T}\right)^{+}}
$$

gives an FS decomposition of $\left(K-S_{T}\right)^{+}$. Since $L^{\left(K-S_{T}\right)^{+}}$is a $\mathbb{P}$-martingale with $L_{T}^{\left(K-S_{T}\right)^{+}} \in L^{2}(\mathbb{P})$, we have only to show the orthogonality of $L^{\left(K-S_{T}\right)^{+}}$to $M$. Since $\left(K-S_{T}\right)^{+} \in \mathbb{D}^{0}$ from Proposition 3.1, we have, by Propositions 3.3 and 3.1 .

$$
\begin{aligned}
\left(K-S_{T}\right)^{+}= & \mathbb{E}_{\mathbb{P}^{*}}\left[\left(K-S_{T}\right)^{+}\right]+\int_{0}^{T} \mathbb{E}_{\mathbb{P}^{*}}\left[D_{t}^{0}\left(K-S_{T}\right)^{+} \mid \mathcal{F}_{t-}\right] d W_{t}^{\mathbb{P}^{*}} \\
& +\int_{0}^{T} \int_{0}^{\infty} \psi_{t, x} \widetilde{N}(d t, d x) \\
= & \mathbb{E}_{\mathbb{P}^{*}}\left[\left(K-S_{T}\right)^{+}\right]-\int_{0}^{T} \mathbb{E}_{\mathbb{P}^{*}}\left[\mathbf{1}_{\left\{S_{T}<K\right\}} S_{T} \mid \mathcal{F}_{t-}\right] \sigma_{t} d W_{t}^{\mathbb{P}^{*}} \\
& +\int_{0}^{T} \int_{0}^{\infty} \psi_{t, x} \widetilde{N}(d t, d x) \\
= & \mathbb{E}_{\mathbb{P}^{*}}\left[\left(K-S_{T}\right)^{+}\right]+\int_{0}^{T} \zeta_{t} d S_{t}+\int_{0}^{T} \int_{0}^{\infty} \psi_{t, x} \widetilde{N}(d t, d x)
\end{aligned}
$$

for some predictable process $\psi \in L^{2}\left(m \times v \times \mathbb{P}^{*}\right)$, which means $L_{t}^{\left(K-S_{T}\right)^{+}}=$ $\int_{0}^{t} \int_{0}^{\infty} \psi_{s, x} \widetilde{N}(d s, d x)$ for any $t \in[0, T]$. Thus, $L^{\left(K-S_{T}\right)^{+}}$is orthogonal to $M$.

By the put-call parity, the following holds:

Corollary 3.6 LRM for call option $\left(S_{T}-K\right)^{+}$is given as $\xi^{\left(S_{T}-K\right)^{+}}=1+\xi^{\left(K-S_{T}\right)^{+}}$. 


\section{Conclusions}

We give representations of LRM of call and put options for BNS models with constraint $\rho=0$. Compared with [1], we relax the restriction on $\beta$; and restrict $\rho$ to 0 instead. The representation (3.4) in Theorem 3.5 coincides with (3.1) in Theorem 3.1 of [1] by substituting 0 for $\rho$. Note that $\beta$ does not appear in representations of LRM, although the density of the MMM is depending on $\beta$.

Some important problems related to LRM for BNS models still remains to future research: development of numerical scheme, comparison with delta hedge, extensions to the fully general case of BNS models, and so forth.

\section{Acknowledgements}

The author would like to thank to Jean-Pierre Fouque for fruitful discussion; and acknowledge the financial support by Ishii memorial securities research promotion foundation.

\section{References}

[1] Arai, T., Suzuki, R.: Local risk minimization for BarndorffNielsen and Shephard models. submitted. Available at http://arxiv.org/pdf/1503.08589v1

[2] Barndorff-Nielsen, O.E., Shephard, N.: Modelling by Lévy processes for financial econometrics. In: Barndorff-Nielsen, O.E., Mikosch,T., Resnick, S. (eds.): Lévy processes -Theory and Applications, pp. 283-318. Birkhäuser, Basel (2001)

[3] Barndorff-Nielsen, O.E., Shephard, N.: Non-Gaussian OrnsteinUhlenbeck based models and some of their uses in financial econometrics. J.R. Statistic. Soc. 63, 167-241 (2001)

[4] Cont R., Tankov P., Financial Modelling with Jump Processes. Chapman \& Hall, London (2004)

[5] Ishikawa, Y.: Stochastic Calculus of Variations for Jump Processes. Walter De Gruyter, Berlin (2013)

[6] Nicolato, E., Venardos, E.: Option Pricing in Stochastic Volatility Models of the Ornstein-Uhlenbeck type. Math. Finance. 13 (4), 445-466 (2003)

[7] Petrou, E. Malliavin calculus in Lévy spaces and applications to finance. Electronic Journal of Probability. 27, 852-879 (2008)

[8] Renaud, J.F.: Calcul de Malliavin, processus de Lévy et applications en finance: quelques contributions. Dissertation, Université de Montréal (2007) Available at http://neumann.hec.ca/pages/bruno.remillard/Theses/JFRenaud.pdf 
[9] Schoutens, W.: Lévy Processes in Finance: Pricing Financial Derivatives. John Wiley \& Sons, Hoboken (2003)

[10] Schweizer, M.: A Guided Tour through Quadratic Hedging Approaches. In: Jouini, E., Cvitanić, J., Musiela, M. (eds.): Option Pricing, Interest Rates and Risk Management (Handbooks in Mathematical Finance), pp. 538574. Cambridge University Press, Cambridge (2001)

[11] Schweizer, M.: Local Risk-Minimization for Multidimensional Assets and Payment Streams. Banach Center Publ. 83, 213-229 (2008)

[12] Solé, J.L., Utzet, F., Vives, J.: Canonical Lévy process and Malliavin calculus. Stochastic Process. Appl. 117, 165-187 (2007)

[13] Wang, W., Qian,L. and Wang, W.: Hedging strategy for unit-linked life insurance contracts in stochastic volatility models. WSEAS Transactions on Mathematic, Volume 12, Issue 4, (2013) 